\title{
Design of a Digital Triaxial Force Sensor for Plantar Load Measurements
}

\author{
Lefan Wang ${ }^{1}$, Dominic Jones ${ }^{1}$, Graham J Chapman ${ }^{2}$, Heidi J Siddle ${ }^{3,4}$, David A Russell ${ }^{3,5}$, Ali Alazmani ${ }^{1}$, and Peter Culmer ${ }^{1}$ \\ ${ }^{1}$ School of Mechanical Engineering, University of Leeds, Leeds LS2 9JT, UK \\ ${ }^{2}$ School of Health Sciences, University of Central Lancashire, Preston PR1 2HE, UK \\ ${ }^{3}$ School of Medicine, University of Leeds, Leeds LS2 9JT, UK \\ ${ }^{4}$ Podiatry Department, Leeds Teaching Hospitals NHS Trust, Leeds LS2 9NL, UK \\ ${ }^{5}$ Leeds Diabetes Limb Salvage Service, Leeds Teaching Hospitals NHS Trust, Leeds LS9 7TF, UK
}

\begin{abstract}
Measurement of load information on the plantar (lower) surface of the foot can provide valuable insights to help identify pathologies like diabetic foot ulcers. Studies have shown that both plantar pressure and shear stress play an important role in foot disorders, especially ulcer formation. However, in this context shear stress is much less studied in comparison with pressure distribution, mainly due to the lack of reliable measurement technologies. In this paper, we propose a triaxial force sensor for measuring plantar loading. The sensor consists of an array of sensing coils combined with an elastomeric spacer and a conductive target. Under loading, the sensor demonstrates differential variations in inductance which are digitized by builtin conditioning circuitry and decoupled. A 3D finite element (FE) model was developed for the system as a design tool. This was validated experimentally and demonstrated a high agreement to the results. In experimental evaluation with multiaxial loading the sensor showed precise operation over the operating range (RMSE: $0.05 \mathrm{~N}$ for shear $(-1.5 \mathrm{~N}-1.5 \mathrm{~N})$ and $0.70 \mathrm{~N}$ for normal force (0-13 N) measurements). The FE model was then used to investigate the effect of undesirable tilting of the target. The results indicated that it is important to minimize the tilting of the target for robust operation in real-world scenarios.
\end{abstract}

Keywords-3D Finite element modelling; digital triaxial sensor; plantar pressure distribution; plantar shear stress.

\section{INTRODUCTION}

Measurements of plantar load distribution (that on the lower surface of the foot) can provide valuable insights into a variety of biomechanical and neurological disorders affecting the lower limb (for example diabetic foot ulcers) [1], [2], illuminate gait asymmetries [3], [4], and assist in supportive footwear design [5]-[7]. Various sensing technologies have been proposed to monitor plantar pressure (which acts perpendicular to the plantar surface, denoted $\sigma_{\text {ver }}$ in Fig. 1(a)). In contrast, plantar shear stress (acting tangential to the plantar surface, shown as $\sigma_{\mathrm{ap}}$ and $\sigma_{\mathrm{ml}}$ in Fig. 1(a)) is far less studied despite it being strongly linked to the development of attributes like plantar callus and ulceration [8], [9]. This is primarily due to the lack of available measurement tools; no commercial systems are capable of plantar shear stress measurement and few research systems exist [7], [10], [11]. Consequently, there is a need for research into multiaxial sensor systems which can measure pressure and shear stress across the plantar surface of the foot.

This work was supported by the UK EPSRC under Grant EP/R041776/1.
In this paper, we report on a digital triaxial force sensor based on inductive coils. The sensing mechanism builds on prior work [12], [13], introducing an integrated low-profile design with improved sensor performance and digital output. The digital output improves noise immunity in the harsh plantar environment and reduce wiring complexity in its intended application where arrays of sensors are embedded within an insole for plantar load measurements, as illustrated in Fig. 1(a). In this context, our key aims in the design process were firstly to achieve a compact sensor footprint while maintaining an appropriate sensor performance for plantar load. Additionally, the foot's plantar surface bends during gait which may act to induce tilting of the compliant sandwich-structured sensors $(\theta)$, as shown in Fig. 1(b). This may adversely affect measurement accuracy and therefore should be investigated to inform future use in clinical applications.
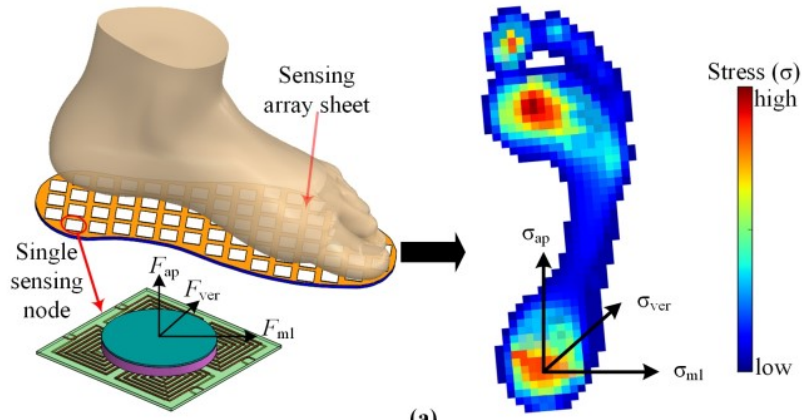

(a)

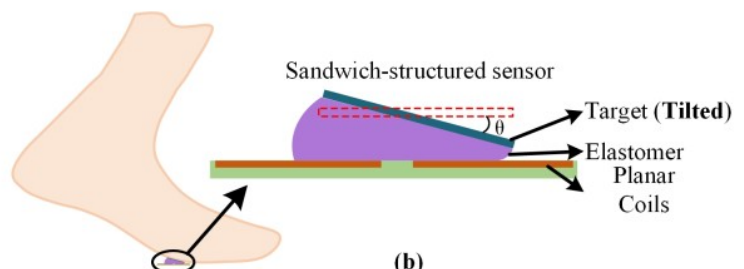

(b)

Fig. 1. (a) Illustration of an insole embedded with an array of triaxial sensing nodes for plantar load measurements; (b) potential tilting of the sensor elements during gait cycles.

\section{SENSOR DESIGN}

\section{A. Operating Principle}


As shown in Fig. 2, the sensor consists of a conductive target supported by an elastomeric spacer on a four-layer flexible printed circuit board (FPCB). The FPCB integrates four 3-layer inductive square coils $\left(L_{0}-L_{3}\right)$, a 4-channel inductance-to-digital converter (LDC1614, Texas Instruments) and conditioning circuitry. This approach minimises the distance between coils and digitising circuitry to avoid noise and parasitic impedances associated with long wire traces.

When a pure vertical load is applied $\left(F_{z}\right)$, the target is brought closer to the coils via the deformation of the elastomer, causing the magnetic coupling between the target and each coil to increase and thus leading to reduction in the inductances of all four coils (see Fig. 2(b)). When the load includes a shear component in the x-axis $\left(F_{x}\right), L_{0}$ and $L_{1}$ decrease whilst $L_{2}$ and $L_{3}$ increase. The same effect applies with the applied shear $F_{y}$.

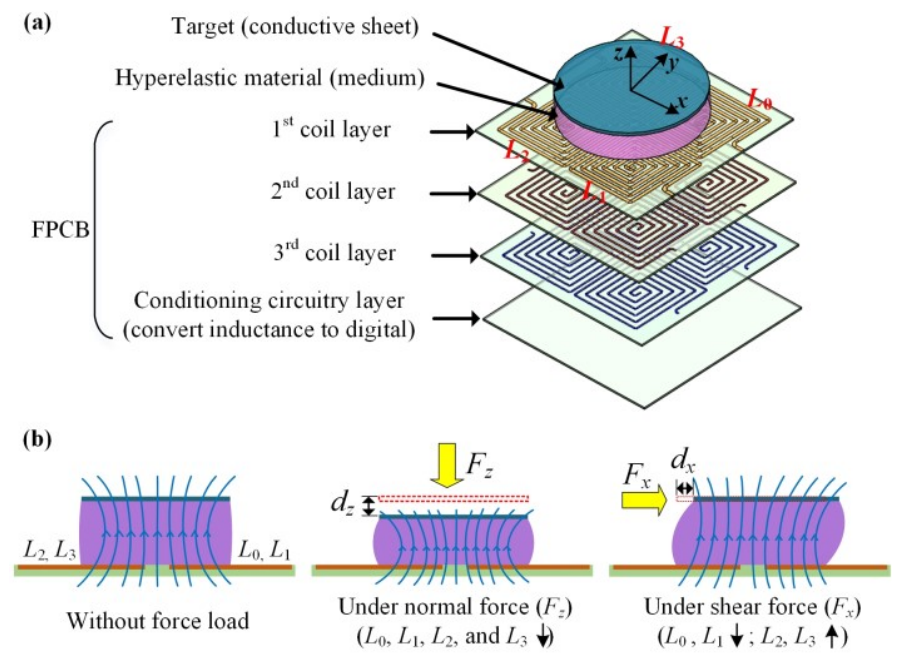

Fig. 2. (a) Sensor structure; (b) Sensing mechanism of the inductive triaxial force sensor.

The sensor's output measurement was determined from the measured inductances by decoupling these elements using a simplified approach (see equation (1)) [13].

$$
\begin{aligned}
& L_{x}=-\Delta L_{1}-\Delta L_{2}+\Delta L_{3}+\Delta L_{4} \\
& L_{y}=-\Delta L_{1}+\Delta L_{2}+\Delta L_{3}-\Delta L_{4} \\
& L_{z}=\left(\Delta L_{1}+\Delta L_{2}+\Delta L_{3}+\Delta L_{4}\right) / 4
\end{aligned}
$$

Where $\Delta L_{i}$ represents the inductance change of Coil $i$ with respect to the coil's free space inductance $L_{\text {free }}$.

\section{B. Sensor Prototype}

A sensor prototype with an overall dimension of $14 \mathrm{~mm} \times$ $14 \mathrm{~mm} \times 0.32 \mathrm{~mm}$ was developed. As shown in Fig. 3, the four square coils (consisting of three layers, $0.1 \mathrm{~mm}$ trace width, 0.1 $\mathrm{mm}$ pitch, and $0.035 \mathrm{~mm}$ copper thickness) were arranged symmetrically in a sensing area of $10 \mathrm{~mm} \times 10 \mathrm{~mm}$. The conditioning circuitry was located on the bottom layer of the FPCB. The conductive target was formed from a circular aluminium film (diameter $8.0 \mathrm{~mm}$, thickness $0.2 \mathrm{~mm}$ ). The elastomeric spacer was laser cut from a 2-mm thick silicon sheet (Ecoflex 20, Smooth-On Inc., USA). The spacer was glued to the target and FPCB using cyanoacrylate with their centre points aligned using locating features (see Fig. 3(a)).

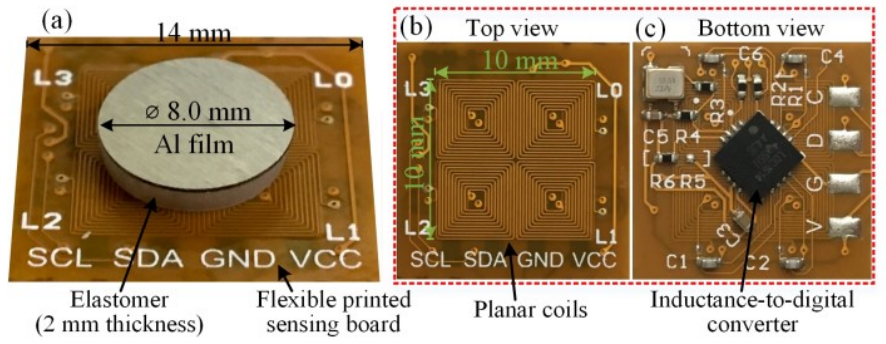

Fig. 3. (a) Integrated triaxial sensor with top and bottom views (b-c).

\section{SENSOR PERFORMANCE EVALUATION}

To evaluate sensor performance we first characterized the response of the sensing coils to movement of the target, then extended this to measurement of load. Finally we explored the effect of target tilting using a 3D finite element (FE) model.

\section{A. Experimental Setup}

A custom measurement system was used to apply multiaxis loading to the sensor. This is based on three linear motorized translation stages (MTS50-Z8, Thorlabs Inc.), shown in Fig. 4. The system was used to apply position-controlled input to the sensor, regulated with motor controllers (KDC101, Thorlabs Inc.) and using a 6-axis load sensor (Nano25, ATI Industrial Automation Inc.,) to measure the applied load.

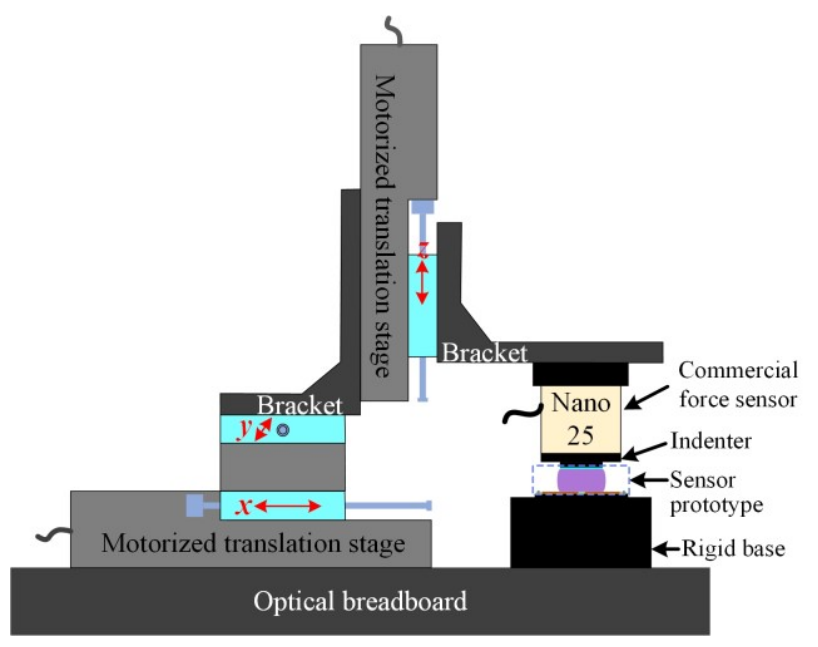

Fig. 4. Three-axis measurement setup.

\section{B. Validation of Sensing Coils' Inductive Responses}

To characterise the inductive coils we used the calibration system to sweep the target through a series of XYZ positions relative to the sensing coils. At every position the inductance of each sensing coil was determined using the integrated digital measurement circuity. The resultant decoupled sensor output $\left(L_{x}, L_{y}\right.$, and $\left.L_{z}\right)$ was then computed using (1) and are presented in Fig. 5. The results show that the sensor is capable of precise measurement within this target displacement range although the response is non-linear at the extremes of movement.

The electromagnetic response of the sensor system was also simulated using a full 3D model (which allows consideration of non-symmetric load and coil configurations) using ANSYS. The same input conditions were used in the model and the outputs were compared to the experimental 
results for validation. From Fig. 5 it is evident that the 3D FE model results were consistent with the experimental system, demonstrating deviations less than $0.02 \mathrm{uH}, 0.02 \mathrm{uH}$, and 0.09 $\mathrm{uH}$ for the motion along the $\mathrm{x}-, \mathrm{y}-$, and $\mathrm{z}$-axis, respectively, providing confidence of the model's fidelity.


Fig. 5. Experimental and simulation response of the sensor to multiaxis target movement.

\section{Sensor Responses to External Force Load}

The calibration rig was then used to characterize the load response of the sensor. A 3D scanning process was performed to obtain the coil inductances under a multiaxis load sweep. To account for the strong crosstalk between axes (evident in Fig. 5), a two-layer feed-forward neural network (15 neurons in the hidden layer) was developed to map the relationship between the output of the four sensing coils and the applied load. The system was evaluated using a new load regime which applied $F_{x}$ and $F_{z}$ simultaneously. The trained network mapped the sensor output into forces which were compared to the directly measured reference loads, shown in Fig. 6.

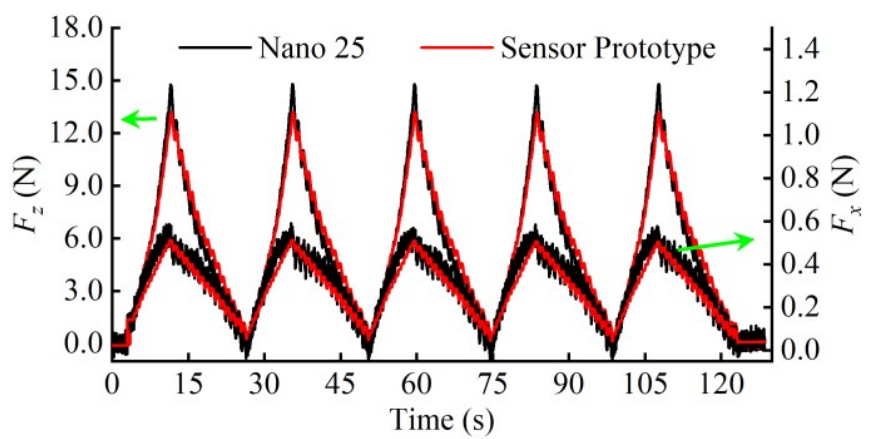

Fig. 6. The calibrated force output of the sensor prototype in comparison with the commercial force sensor Nano 25 .
The response demonstrates a close agreement between the sensor and reference measurements throughout the multiaxial loading, with an RMSE of $0.05 \mathrm{~N}$ for $F_{x / y}$ measurements and $0.70 \mathrm{~N}$ for $F_{z}$ detection. Some discrepancy is noted at points with peak forces, possibly related to the different dynamic response characteristics (e.g. overshoot) between the sensor prototype and the reference Nano25 (silicon strain gauges). Overall, the calibrated sensor prototype shows a promising capability for triaxial force measurements.

\section{Effect of Tilting Target to Sensor Responses}

To characterise the effect of sensor deformation under complex loading we used the FE sensor model to investigate tilting of the target. As shown in Fig. 7, the sensor outputs varied as a function of tilting and to varying degrees across the sensor's range. For lateral motion $\left(d_{z}=1 \mathrm{~mm}\right)$, the inductance changed $11.9 \%$ full scale reading (FS) at $3^{\circ}$ tilt and up to $57.3 \%$ FS under a tilt of $10^{\circ}$ (the physical maximum). Under the vertical movement $\left(d_{x}=d_{y}=0\right)$ the maximum variation in inductance wad $1.6 \%$ at $3^{\circ}$ tilt and $24.1 \%$ FS under $10^{\circ}$ tilt. It is therefore evident that tilting of the target can induce a significant error and thus techniques are required to minimize such effects when used in real-world scenarios.
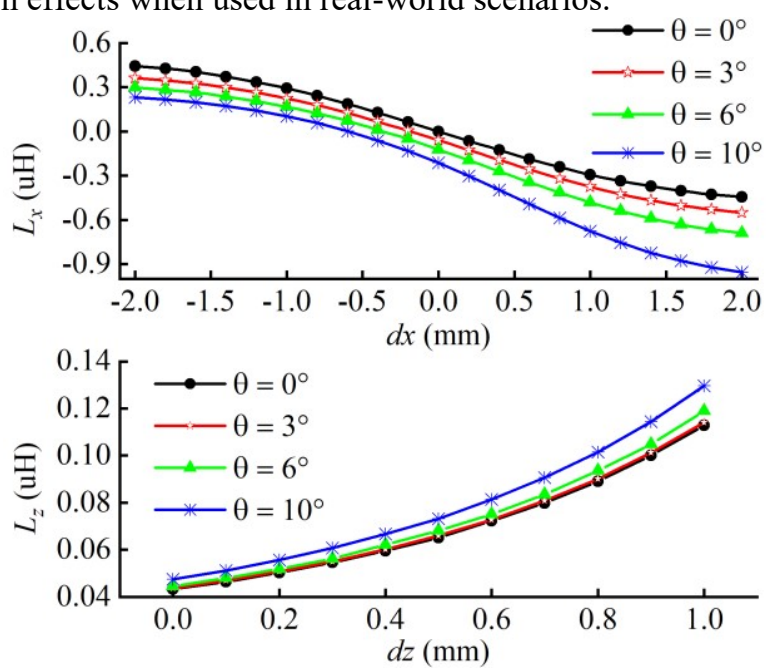

Fig. 7. The effect of the tilting angle of the target on the sensor outputs.

\section{CONCLUSIONS}

In this research, we implemented a compliant digital triaxial force sensor for measuring plantar force load distribution. A 3D simulation model was developed to investigate the sensors response under different displacement conditions using FE method. The sensor performance was also experimentally studied using a triaxial calibration system. The simulation and experimental results were consistent, showing a maximum deviation of $0.02 \mathrm{uH}, 0.02 \mathrm{uH}$, and $0.09 \mathrm{uH}$ for the $\mathrm{x}-, \mathrm{y}-$, and $\mathrm{z}$-axis motion, respectively. To map the relationship between the external force load and the sensor outputs, a two-layer neural network was employed. The calibrated sensor then measured the multiaxial external load with close agreement to a reference loadcell. Under complex loading we observed that the current system can be sensitive to sensor deformation which induces errors. However, ongoing research is investigating how to mitigate theses effects and translate this promising sensing technology toward clinical utility. 


\section{References}

[1] A. K. Buldt, S. Forghany, K. B. Landorf, P. Levinger, G. S. Murley, and H. B. Menz, "Foot posture is associated with plantar pressure during gait: A comparison of normal, planus and cavus feet," Gait Posture, vol. 62, pp. 235-240, May 2018.

[2] H. B. Menz, "Biomechanics of the Ageing Foot and Ankle: A MiniReview," Gerontology, vol. 61, no. 4, pp. 381-388, Nov. 2014.

[3] K. Bosch and D. Rosenbaum, "Gait symmetry improves in childhoodA 4-year follow-up of foot loading data," Gait Posture, vol. 32, no. 4, pp. 464-468, Oct. 2010.

[4] H. P. Becker, D. Rosenbaum, T. Kriese, H. Gerngross, and L. Claes, "Gait asymmetry following successful surgical treatment of ankle fractures in young adults.," Clin. Orthop. Relat. Res., no. 311, pp. $262-$ 9, Feb. 1995.

[5] M. ) Mueller, "Application of Plantar Pressure Assessment in Footwear and Insert Design," 1999.

[6] S. F. E. Praet and J.-W. K. Louwerens, "The influence of shoe design on plantar pressures in neuropathic feet.," Diabetes Care, vol. 26, no. 2, pp. 441-5, Feb. 2003.
[7] A. H. Abdul Razak, A. Zayegh, R. K. Begg, and Y. Wahab, "Foot plantar pressure measurement system: A review," Sensors (Switzerland), vol. 12, no. 7, pp. 9884-9912, 2012.

[8] M. Yavuz et al., "Plantar shear stress in individuals with a history of diabetic foot ulcer: An emerging predictive marker for foot ulceration," Diabetes Care, vol. 40, no. 2, pp. e14-e15, 2017.

[9] D. G. Armstrong, A. J. M. Boulton, and S. A. Bus, "Diabetic Foot Ulcers and Their Recurrence," N. Engl. J. Med., vol. 376, no. 24, pp. 2367-2375, 2017.

[10] J. A. Ramirez-Bautista, J. A. Huerta-Ruelas, S. L. Chaparro-Cárdenas, and A. Hernández-Zavala, "A Review in Detection and Monitoring Gait Disorders Using In-Shoe Plantar Measurement Systems," IEEE Rev. Biomed. Eng., vol. 10, pp. 299-309, 2017.

[11] S. Rajala and J. Lekkala, "Plantar shear stress measurements - A review," Clin. Biomech., vol. 29, no. 5, pp. 475-483, 2014.

[12] L. Du, X. Zhu, and J. Zhe, "An inductive sensor for real-time measurement of plantar normal and shear forces distribution," IEEE Trans. Biomed. Eng., vol. 62, no. 5, pp. 1316-1323, 2015.

[13] H. Wang et al., "Design and Characterization of Tri-axis Soft Inductive Tactile Sensors,” IEEE Sens. J., vol. 18, no. 19, pp. 7793-7801, 2018 